\title{
Análisis socioeconómico de Pueblos Mágicos. El caso de Pinos, Zacatecas, México
}

\section{Socioeconomic analysis of Magical Towns. The case of Pinos, Zacatecas, Mexico}

Josefina Torres de Santiago1; Gilberto Salas Rodríguez²; Armando Flores de la Cruz ${ }^{3}$

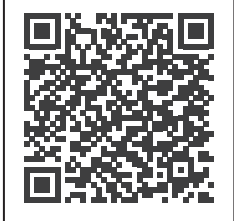

\section{Palabras clave:}

desarrollo regional, economía, economía cultural, pueblo mágico, marketing, turismo.

Artículo de reflexión

Fecha de recepción:

15 de marzo de 2021

Fecha de aprobación:

02 de agosto de 2021

Fecha de publicación:

28 de octubre de 2021

Esta publicación se encuentra bajo licencia:

Creative Commons

Reconocimiento-

NoComercial-SinObraDerivada 4.0 Internacional

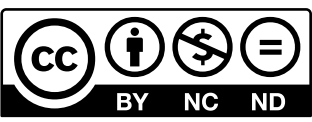

\section{Resumen}

El análisis socioeconómico del Programa Pueblos Mágicos (PPM) en el municipio de Pinos, Zacatecas, México, evidencia los efectos socioeconómicos del PPM. Problemática: en la implementación del PPM en la comunidad se han generado estrategias en el desarrollo turístico económico con beneficios económicos para los empresarios, autoridades y ciudadanos de la comunidad. Objetivo: el presente estudio valida el nivel de contribución del PPM en las actividadeseconómicas y la relación con el turismo en el municipio de Pinos, Zacatecas, México. Materiales y métodos: la indagación tiene el enfoque mixto, cuantitativo y cualitativo. Se entrevistaron a ciudadanos de la comunidad a través de un cuestionario con una muestra representativa de informantes. En el enfoque cualitativo se tuvo la participación de representantes de la autoridad municipal. El análisis se realiza por medio del método descriptivo y causal. Resultados y discusión: en el estudio se constata el desarrollo turístico económico integral y los beneficios de la implementación del

1 Licenciada en Economía. Maestra en Administración. Maestra en Mercadotecnia. Doctora en Proyectos. Universidad Tecnológica del Estado de Zacatecas. División de Administración y Desarrollo de Negocios, México. jtorres@utzac. edu.mx, ORCID: https://orcid.org/0000-0002-8385-7012

2 Licenciado en Administración. Maestro en Administración. División en Desarrollo de Negocios. Universidad Tecnológica del Estado de Zacatecas, México. gsalas@utzac.edu.mx, ORCID: https://orcid.org/0000-0002-1048-1309

3 Ingeniero en Química. Maestro en Administración. División en Desarrollo de Negocios. Universidad Tecnológica del Estado de Zacatecas, México. adelacruz@utzac.edu.mx, ORCID: https://orcid.org/0000-0003-2592-6929 


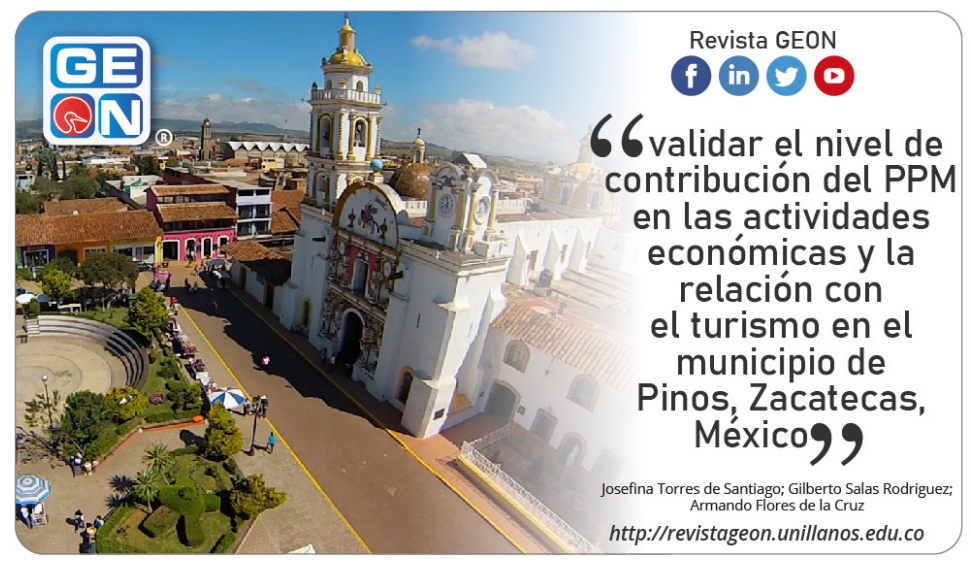

PPM percibidos por los ciudadanos, funcionarios de la autoridad municipal y empresarios en la mejora de la calidad de vida de los pobladores y la contribución de las empresas turísticas en actividades de responsabilidad social y cultural en la comunidad. Conclusiones: en la investigación se constata por medio de los informantes el impacto del desarrollo turístico económico en la generación de empleos, la oferta de productos y servicios turísticos y la valoración y reconocimiento de los recursos naturales y culturales en la comunidad. Contribución: el análisis socioeconómico en la comunidad evidencia el desarrollo turístico económico con la implementación del PPM y el impacto de las actividades del turismo en la comunidad.

Palabras clave: desarrollo regional, economía, economía cultural, pueblo mágico, marketing, turismo.

Códigos JEL: Z320 Turismo y desarrollo; Z33 Turismo: marketing y finanzas; D62 Economía del bienestar

Cómo citar este artículo / Toreference this article:

Torres de Santiago, J., Salas Rodríguez, G., \& Flores de la Cruz, A. (2021). Análisis socioeconómico de Pueblos Mágicos. El caso de Pinos, Zacatecas, México. Revista GEON (Gestión, Organizaciones Y Negocios), 8(2), e-309. https://doi. org/10.22579/23463910.309

\section{Abstract}

The socioeconomic analysis of the Magical Towns Program (PPM) the municipality of Pinos, Zacatecas, Mexico shows the socioeconomic effects of the PPM. Problem: The implementation of the PPM in the community has generated strategies for economic tourism development with economic benefits to entrepreneurs, authorities and citizens 
Cómo citar este artículo / Toreference this article:

Torres de Santiago, J., Salas Rodríguez, G., \& Flores de la Cruz, A. (2021). Análisis socioeconómico de Pueblos Mágicos. El caso de Pinos, Zacatecas, México. Revista GEON (Gestión, Organizaciones Y Negocios), 8(2), e-309. https://doi. org/10.22579/23463910.309 in the community. Objective: To validate the level of contribution of the PPM in economic activities and the relationship with tourism in the municipality of Pinos, Zacatecas, Mexico. Materials and methods: The research has a mixed quantitative and qualitative approach. Citizens of the community were interviewed through a questionnaire with a representative sample of informants. The qualitative approach involved the participation of representatives of the municipal authority. The analysis is carried out by means of the descriptive and causal method. Results and discussion: The study confirms the integral economic tourism development and the benefits with the implementation of the PPM, perceived by the citizens, municipal authority officials, businessmen, in the improvement of the quality of life of the inhabitants and the contribution of the tourism companies in activities of social and cultural responsibility in the community. Conclusion: In the research, the informants confirmed the impact of economic tourism development in the generation of jobs, the offer of tourism products and services, and the valuation and recognition of the resources in the community. It contributes to the socioeconomic analysis in the community evidences the economic tourism development with the implementation of the PPM and the impact of tourism activities in the community.

Keywords: Cultural economics; economy; magic town; marketing; regional development; tourism.

JEL Code: Z320 Tourism and development; Z33 Tourism: marketing and finance; D62 Welfare economics

\section{Introducción}

El Programa Pueblos Mágicos de México (PPM) es conocido por contribuir a revalorar a un conjunto de poblaciones del país en el imaginario colectivo de la nación, con alternativas variadas en la cultura y originalidad turística para los visitantes nacionales y extranjeros. En México existen 132 pueblos mágicos y seis de ellos están ubicados en el estado de Zacatecas (SECTUR, 2014).

A partir de 2012, el municipio de Pinos, Zacatecas, es nombrado Pueblo 
Mágico por la Secretaría de Turismo de México. Este pueblo minero se destaca por sus atractivos turísticos en las exhaciendas mineras y de mezcal, la hacienda La Pendencia, Sierra de Pinos, la Torre del Reloj Público, el Museo de Arte Sacro, la parroquia de San Matías, Cristo del Corazón Flotante, entre otros (DATATUR, 2020).

En la indagación se plantea como objetivo validar el nivel de contribución del PPM en las diversas actividades económicas relacionadas con el turismo en el municipio de Pinos, Zacatecas, México. Esta aproximación evidencia la permanencia del PPM para diferenciar el impacto que se ha tenido en la comunidad.

En el estudio se implementó la metodología a través de técnicas y métodos como la observación, las entrevistas a funcionarios públicos, que representan la autoridad municipal, y una encuesta a los ciudadanos en la comunidad. En el enfoque cuantitativo se entrevistaron a ciudadanos de la comunidad por medio de un cuestionario. En el enfoque cualitativo se tuvo la participación de informantes de la autoridad municipal. El análisis se realizó a través de los métodos descriptivo y causal.

Por consiguiente, los resultados y la discusión de la investigación constatarán el impacto del programa en el desarrollo turístico económico percibido por los ciudadanos y representantes de la autoridad municipal. Los datos que se presentan aluden al reconocimiento y valoración del desarrollo turístico económico equi- librado, así como a la integración de productos y servicios en el fortalecimiento de la actividad turística y sostenible en la comunidad y la región.

Finalmente, se deduce en la investigación que el desarrollo turístico económico evidencia la relación positiva en la generación de empleos, la oferta de productos y servicios turísticos y el desarrollo equitativo e integral en el aprovechamiento sostenible de los recursos naturales por los ciudadanos en la comunidad. La investigación contribuye al desarrollo turístico económico en la permanencia del PPM y el impacto del turismo en la comunidad. Además, se proponen nuevas líneas de investigación ante el contexto socioeconómico y el mercado nacional y extranjero.

\section{Contexto teórico}

En el contexto internacional, el turismo es conocido como un fenómeno económico, social y cultural orientado al desplazamiento de personas a lugares o países que estén fuera del entorno tradicional por diversos motivos: personales, de negocio, culturales o profesionales (OMT, 2020).

De acuerdo con la Organización Mundial de Turismo (OMT, 2020), el turismo sostenible se define como "aquel que pretende satisfacer las necesidades de los turistas y de los destinos turísticos protegiendo e incrementando las oportunidades a futuro".

En México, el turismo sostenible e inclusivo es relevante. De acuerdo con el Programa Nacional de Desarrollo (PND) 2019-2024, en el tema de eco- 
nomía se deriva el Programa Sectorial de Turismo 2020-2024. La política turística está orientada a la inclusión de todos los sectores de la población, respetando los usos, las costumbres y la preservación de los territorios de los pueblos originarios y fomentando la igualdad sustantiva de hombres y mujeres, así como también la dignidad de los adultos mayores y el derecho de los jóvenes a tener un lugar para construir el futuro del país. El modelo de desarrollo turístico que se plantea está enfocado al "[...] respeto de los habitantes y su hábitat equitativo, rectificador de las desigualdades, defensor de las culturas locales y del medio ambiente, perceptivo de las formas regionales y locales de la economía y atento a los habitantes futuros del país" (SECTUR, 2020).

Las aportaciones de Peniche et al. (2017) en el modelo de turismo están orientadas a la inclusión de la protección de las reservas naturales de los países receptores y al fomento del desarrollo económico de los actores locales y de la comunidad y su actividad productiva. Ante el panorama presentado, la actividad turística se percibe como los actos que llevan a cabo las personas para que sucedan hechos de carácter turístico-recreacional, con la colaboración de las empresas y organizaciones que invierten los recursos para la producción de bienes y servicios en beneficio de las comunidades con patrimonio turístico. Se destaca que la importancia de los atractivos turísticos, los valores, la naturaleza, la cultura o el sitio influyen en la motivación de la afluencia de la población foránea (SECTUR, 2020).
Para esta investigación, es relevante el enfoque de la calidad de la ubicación desde tres dimensiones:

[...] qué hay en referencia al entorno físico; quién hay en relación con las personas y, finalmente, qué pasa en relación con la vitalidad de la vida en la calle y con las actividades sociales y culturales realizadas que generan un amplio abanico de opciones para diferentes personas en distintos momentos de sus vidas (Romero et al., 2020).

En resumen, en la extensa literatura se percibe conceptualmente al turismo cultural como "[...] un tipo de actividad turística expresada por la motivación principal del visitante en la ilustración, apreciación, en descubrir y consumir las atracciones, productos culturales tangibles e intangibles en un destino turístico" (Espeso, 2019).

Otra de las aportaciones conceptuales de Parkin (2018) es la de economía como "[...] la ciencia social que estudia las elecciones que hacen los individuos, las empresas, los gobiernos y las sociedades en su conjunto para enfrentar la escasez". Esta aportación teórica nos aproxima a dimensionar la importancia de las actividades económicas para la sociedad. Por consiguiente, la actividad productiva de los empresarios se vincula con el grado de diversidad cultural expresado por la riqueza natural de la comunidad. En resumen, la cultura es favorable para el desarrollo económico y la innovación (Facchini, 2020).

En este estudio es relevante la teoría conceptual de marketing en la orienta- 
ción en marketing turístico, según las aportaciones de Kotler y Armstrang (2017), como la actividad "encaminada a atraer clientes y administrar relaciones redituables, nuevos clientes y ofrecerles un valor superior, en la satisfacción de sus necesidades". Las tendencias de ventas digitales y automáticas nos permiten, además, definir el marketing digital como la aplicación de las estrategias de comercialización llevadas a cabo en los medios digitales con los beneficios de interacción, análisis y medición, alcance a la audiencia, personalización y precisión, bajos costos de operación, flexibilidad, competitividad y retención de clientes con el uso de la tecnología en realidad virtual y aumentada, y por el uso de herramientas tecnológicas (RDSTATION, 2020).

Las aportaciones teóricas en el desarrollo turístico económico y en el uso de las herramientas tecnológicas como táctica en la generación de un mayor impacto en el consumidor-turista a través de la promoción de destinos turísticos es la estrategia para el posicionamiento y conocimiento del atractivo turístico a nivel nacional e internacional (Sandoval et al., 2021, p. 295).

En el enfoque conceptual del PPM, derivado de políticas públicas a nivel nacional, la ventaja competitiva en los Pueblos Mágicos es la expresión cultural de las comunidades que históricamente, y ante la evolución, han preservado, valorado y protegido el patrimonio cultural y natural, así como el legado histórico, que son reconocidos en la generación de la riqueza de México (Hofmann, 2015).
El Pueblo Mágico nos aproxima a identificarlo como "[...] una localidad que tiene atributos simbólicos, leyendas, historia, hechos trascendentes, cotidianidad y magia que emanan en cada una de sus manifestaciones socioculturales, y que significan una gran oportunidad para el aprovechamiento turístico" (SECTUR, 2014). La Secretaría de Turismo implementa el Programa de Pueblos Mágicos para la revaloración del conjunto de poblaciones del país que han estado en el imaginario colectivo de la nación y que representan alternativas diferentes para los visitantes nacionales y extranjeros (SECTUR, 2020).

Finalmente, estas aportaciones teóricas y de contexto nos permiten generar un acercamiento al estudio del análisis socioeconómico de Pueblos Mágicos y su impacto en el mejor nivel de vida de la población, así como en el desarrollo turístico económico de la comunidad y de la región.

\section{Materiales y métodos}

En esta investigación se diseñó una encuesta con el enfoque mixto (Hernández et al., 2014). Las técnicas utilizadas fueron consultas de estudios, documentos, datos estadísticos de SECTUR e INEGI-DENUE, observación y encuesta. En el enfoque cualitativo se tuvo la participación de 10 informantes de la autoridad municipal a través de una encuesta de 12 ítems. El análisis se realizó por medio del método descriptivo y causal. En el enfoque cuantitativo se entrevistaron a ciudadanos de la comunidad a través de un cuestionario. El proceso 
fue de tipo probabilístico, destacando las características de la población y del muestreo aleatorio simple, con la muestra representativa de 360 . El diseño de la encuesta tuvo 20 ítems, las escalas fueron de tipo opción múltiple, rangos y likert, con la finalidad de facilitar a los entrevistados (Fisher \& Espejo, 2017).

La validez del instrumento de medición se realiza, según la aportación de Hernández et al., (2014), con la validez de contenido, de criterio y de constructo. La fiabilidad constituye una de las más importantes cualidades técnicas de los instrumentos de medida, nos indica la precisión y constancia en la información recogida, lo que resulta indispensable para hacer investigaciones con un nivel de rigor aceptable. El índice alfa de Cronbach fue del 0,90, con una alta consistencia de confiabilidad de los ítems que resultó aceptable.

En resumen, a través de la metodología se definen las formas de recolección de datos, trabajo de campo y tabulación para la interpretación y análisis de los datos. A continuación, se presentan los resultados de la investigación.

\section{Resultados}

En esta sección se presenta el análisis de los datos de la investigación con las características de los sujetos de estudio que proporcionaron respuesta a los instrumentos, mediante la encuesta dirigida. En el enfoque cualitativo se entrevistaron a 10 funcionarios del gobierno municipal. De acuerdo con el análisis de los datos, el 90\% de los informantes han realizado estudios de licenciatura y posgrado, por lo que ocupan los puestos de director, jefe de área y regidor de turismo. En la variable de infraestructura de los servicios de agua potable, drenaje, alcantarillado, mercados, limpieza, alumbrado público, entre otros, el 100\% de los informantes mencionó que estos se mejoraron. En la variable de la inversión pública, es evidente la creación de nuevos empleos en la comunidad; el $40 \%$ de los informantes mencionó que se destinó del 21\% a más del 50\% al turismo en los últimos años. En el seguimiento al trato recibido a partir del nombramiento de Pueblo Mágico, el $80 \%$ de los informantes señaló que las instituciones y dependencias del Gobierno estatal y federal ofrecieron un trato especial a la comunidad por el reconocimiento de Pueblo Mágico. En resumen, las autoridades del Gobierno municipal calificaron la calidad de los servicios en la comunidad en las categorías de buena y excelente, con el $80 \%$ de los informantes.

En el enfoque cuantitativo, los datos en la indagación evidencian la relevancia de las actividades del turismo en el desarrollo económico y social en el municipio de Pinos, Zacatecas. El perfil de los informantes expresa la participación de los hombres con el 56\% y de las mujeres con el 44\%. El rango de edad de 15 a 29 años representa la mayoría, el 70\% de la población joven, sujeta de estudio en esta investigación; el nivel educativo más representativo es de licenciatura y posgrado con el 53\%, preparatoria con el 38\%, primaria y secundaria con el $7 \%$ y otros estudios con el 2\% (tabla 1). 
De acuerdo con el comportamiento de los datos de la edad y el nivel educativo, se identifica que la mayoría son jóvenes y el nivel educativo es la licenciatura y el posgrado con el 53\% (figura 1).

Tabla 1.Datos generales de los informantes por sexo, edad y escolaridad (2020)

\begin{tabular}{|c|c|c|c|}
\hline \multirow{4}{*}{ Sexo } & & Frecuencia & $\%$ \\
\hline & Femenino & 157 & $44 \%$ \\
\hline & Masculino & 203 & $56 \%$ \\
\hline & Total & 360 & $100 \%$ \\
\hline \multirow{6}{*}{ Edad } & $15-19$ & 8 & $2 \%$ \\
\hline & 20-29 años & 243 & $68 \%$ \\
\hline & $30-39$ & 82 & $23 \%$ \\
\hline & $40-49$ & 20 & $6 \%$ \\
\hline & más de 50 años & 7 & $2 \%$ \\
\hline & Total & 360 & $100 \%$ \\
\hline \multirow{5}{*}{ Escolaridad } & Primaria y secundaria & 25 & $7 \%$ \\
\hline & Preparatoria & 138 & $38 \%$ \\
\hline & Licenciatura y posgrado & 189 & $53 \%$ \\
\hline & Otros estudios & 8 & $2 \%$ \\
\hline & Total & 360 & $100 \%$ \\
\hline
\end{tabular}

Fuente: elaboración propia de los autores.

Figura 1. Datos generales de los informantes por sexo, edad y escolaridad (2020).

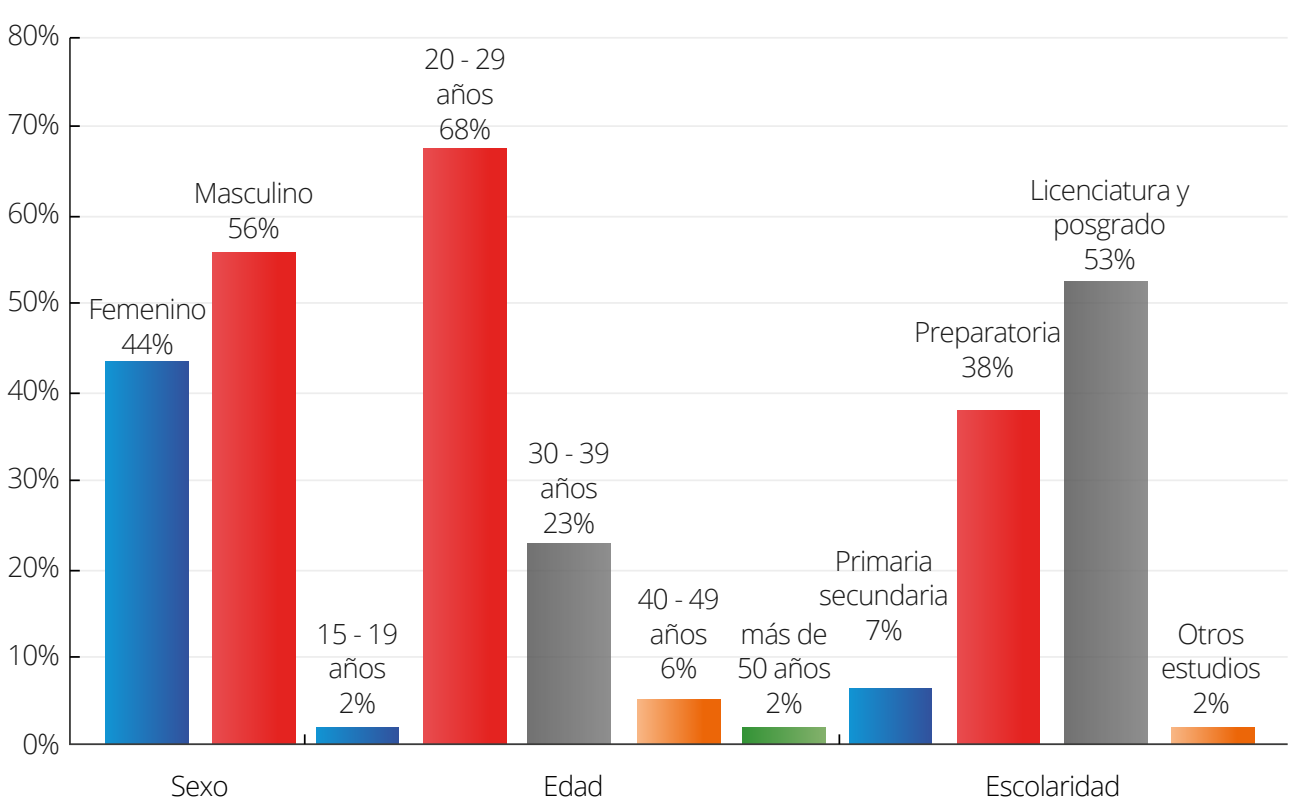

Fuente: elaboración propia de los autores. 
En el desarrollo regional, de acuerdo con la indagación que se presenta, las principales actividades económicas en la comunidad relacionadas con el turismo, en la categoría de alto interés, son el comercio y los servicios con el 49\%; la agricultura y la ganadería con el 37\%; la industria con el $5 \%$ y la pesca con el 2\%. En la comunidad de Pinos se ha desarrollado el comercio y los servicios a través de los servicios de hotelería, el transporte, los recorridos turísticos y el comercio de artesanías tradicionales elaboradas en los talleres con barro, como jarros, platos, cazuelas, entre otros. En el 5\% de la industria es relevante la agroindustria, destacando la tuna y el Mezcal de la Pendencia, así como su historia en las haciendas mineras (figura 2). Así mismo, es relevante el desa- rrollo turístico económico generado a partir del nombramiento de Pueblo Mágico antes de la pandemia y pospandemia, y la implementación de plataformas tecnológicas y social media como herramientas para la promoción turística por parte de los sectores públicos y privados.

De acuerdo con la valoración económica de la comunidad, a partir del nombramiento de Pueblo Mágico, el $26,4 \%$ de la ciudadanía la percibe como buena, el 3,3\% como muy buena, el $58,1 \%$ como regular y el $4,7 \%$ sin cambio (figura 3). Es evidente que la mayoría de los informantes de la comunidad identificaron un cambio económico en las diversas actividades al ofrecer los productos y servicios turísticos al mercado nacional e internacional.

Figura 2. Interés de la comunidad para colaborar en actividades asociadas al turismo por sector económico.

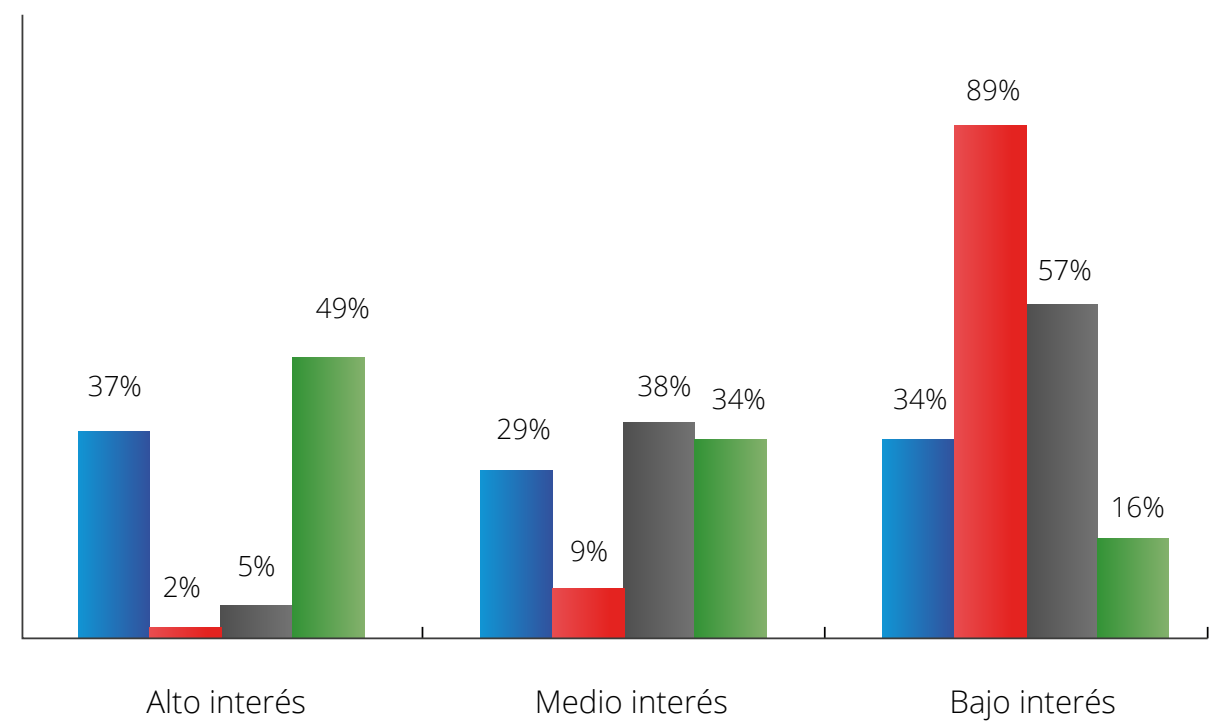

Agricultura / ganadería —Pesca —Industria Comercio / servicios

Fuente: elaboración propia de los autores. 
Figura 3. Valoración económica de la comunidad a partir del nombramiento de Pueblo Mágico.

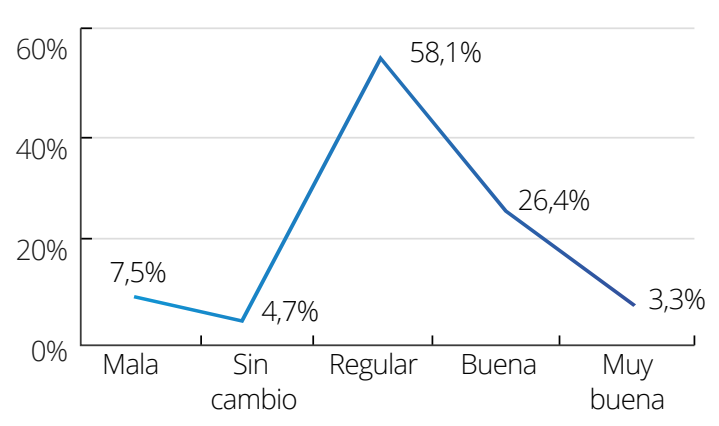

Fuente: elaboración propia de los autores.

Los sectores sociales de la comunidad perciben ser beneficiados por las actividades del turismo: las autoridades con el 40\%; los empresarios y comerciantes con el 32\%; finalmente, toda la comunidad con el 28\%. En resumen, el impacto del turismo ha generado desarrollo económico en los sectores de la sociedad (tabla 2).

Tabla 2.Sectores sociales con beneficio del turismo en la comunidad (2020)

\begin{tabular}{l|c|c}
\multicolumn{1}{c|}{ Sector social } & N & \% \\
\hline Empresarios y comerciantes & 116 & $32 . \%$ \\
\hline Autoridades & 145 & $40 \%$ \\
\hline Toda la comunidad & 99 & $28 \%$ \\
Total & $\mathbf{3 6 0}$ & $\mathbf{1 0 0 \%}$ \\
\hline
\end{tabular}

Fuente: elaboración propia de los autores.

La inversión en el turismo antes de la pandemia fue relevante gracias a la creación de productos y servicios por los empresarios de la localidad. La mayoría estuvo representada por el financiamiento del turismo: el 67\% por empresarios de origen interno y el 33\% por empresarios de origen externo (tabla 3).
Tabla 3. Financiamiento del turismo (2020)

\begin{tabular}{lc}
\multicolumn{1}{c}{ Origen de la inversión } & \% \\
\hline Internos & 67 \\
\hline Externos & 33 \\
Total & $\mathbf{1 0 0}$ \\
\hline
\end{tabular}

Fuente: elaboración propia de los autores.

Las empresas turísticas en la región identificaron áreas de oportunidad para proyectos de productos y servicios en el turismo, entre los que se incluyen las artesanías de barro, los servicios turísticos de recorridos en sitios de esparcimiento y diversión, los servicios de guías, el comercio turístico, el alojamiento, los alimentos y las bebidas y el transporte.

Al indagar por el comportamiento del turista en la comunidad, el 94\% de los informantes percibe que el turista tuvo un comportamiento positivo en el respeto a las tradiciones, costumbres y recursos de la comunidad, mientras que el $6 \%$ considera que lo contrario (figura 4). En cuanto a los recursos importantes y valorados por la comunidad, los recursos de monumentos históricos son los más importantes y los más valorados, con el $86 \%$ y el $82 \%$, respectivamente.

Finalmente, se observa la relevancia del turismo cultural en la región en la contribución a la generación de empleo temporal y permanente en la población local; en identificar la mejora en la calidad de vida de la población en el municipio de Pinos, Zacatecas, percibida por los ciudadanos y en fomentar las actividades económicas (Pérez, et al., 2017) relacionadas con el turismo, con impacto en la economía 
en cuanto al crecimiento del empleo temporal y permanente. Estas variables están insertadas en un modelo sostenible e incluyente del turismo.

Figura 4. Comportamiento positivo del visitante en el Pueblo Mágico de Pinos, Zacatecas.

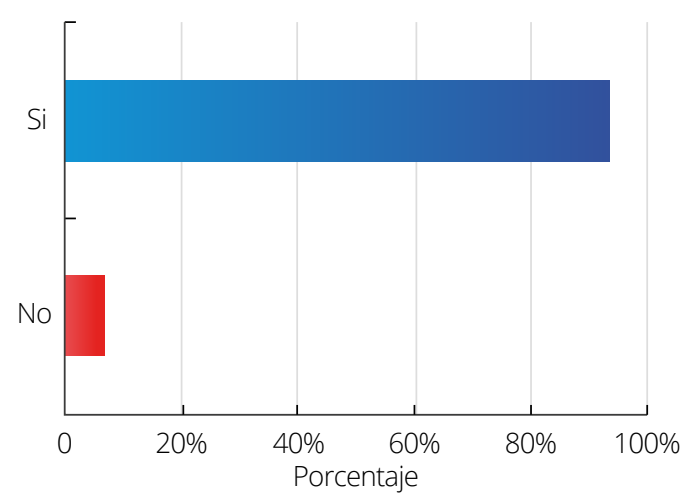

Fuente: elaboración propia de los autores.

\section{Discusión}

El municipio de Pinos, Zacatecas, se ubica a 125 kilómetros al sureste de la capital del estado de Zacatecas, cuenta con una superficie de 3168,6 km2, la población total es de 72.241, la edad mediana, es decir, la mitad de la población tiene 26 años o menos, la densidad de población es de 22,8 habitantes por km2, la población económicamente activa es del 51,3\%, (INEGI, 2020). Según el Consejo Nacional de Población (CONAPO, 2020), Pinos cuenta con un grado de marginación medio, el índice de marginación (IM) es 54.234, el porcentaje de la población ocupada con ingresos menores a dos salarios mínimos es del 80,45\%, el lugar que ocupa en el contexto nacional es el 1191. Las actividades económicas principales son la agricultura, el comercio, el turismo cultural y la gastronomía típica de la comunidad.

De acuerdo con los resultados, Pinos, Zacatecas, tiene características únicas como Pueblo Mágico a partir del nombramiento en 2012. El desarrollo turístico económico es percibido por los informantes, ciudadanos y autoridades municipales como detonante en la comunidad. Se destaca, además, el reconocimiento de marca de Pueblo Mágico que contribuye al desarrollo turístico económico de las diversas actividades culturales y económicas. Por consiguiente, se evidencia el impacto social y económico del PPM en la comunidad, en la mejora en la calidad de vida, en la generación de empleos y en el financiamiento en los sectores productivos por parte de empresarios de la región (SECTUR, SECTURZ, UAZ, 2014).

Ciertamente, ante el cambio del contexto durante el análisis y la discusión, la permanencia del PPM contribuye a la mejora de la calidad de vida de la población en sus tres dimensiones: el entorno físico, las personas y la vitalidad que se percibe tanto en la calle como en las actividades sociales y culturales (Romero et al., 2020) y el enfoque tecnológico que facilita la promoción y el desarrollo turístico sostenible e incluyente en la comunidad (Orellana, 2020).

Por consiguiente, el turismo cultural, relacionado con las actividades económicas, ha adquirido un crecimiento importante en las "dinámicas sociales, culturales, medioambientales y eco- 
nómicas con efectos positivos" (Cayo \& Apaza 2017). Evidentemente, la promoción de destinos turísticos se ha caracterizado como la estrategia imprescindible para el posicionamiento y conocimiento del atractivo turístico a nivel nacional e internacional, con el uso de herramientas tecnologías innovadoras que generan un mayor impacto en el consumidor (Sandoval et al., 2021).

\section{Conclusiones}

Esta indagación contribuye a generar una metodología innovadora que evidencie el desarrollo turístico económico en la comunidad y, por consiguiente, se fortalezcan los programas derivados de la implementación de políticas públicas en el turismo cultural y en las actividades económicas relacionadas con el turismo, con la orientación hacia el impacto económico y social en el sureste de Zacatecas por el reconocimiento de marca de Pueblos Mágicos, posicionado a nivel local, regional, estatal, nacional y global en el entorno (Rodríguez \& San-Martin, 2020).

Por lo tanto, en el estudio se constata el efecto causal en la relación positiva en generación de empleos, inversión y mejora en la calidad de vida de los ciudadanos y la autoridad municipal en la comunidad (SECTUR, 2020). Esto se refleja en el desarrollo turístico económico, el reconocimiento turístico y cultural, el reconocimiento de marca, la colaboración de las instancias gubernamentales en los diferentes ámbitos, los ciudadanos, empresarios en la innovación de la oferta y demanda turística, los servicios públicos, entre otros (Torres, 2020). Los informantes perciben el reconocimiento de marca desde la llegada del turista, con un enfoque positivo, con respeto a las tradiciones y costumbres que se ofrecen en el municipio y con la contribución de las empresas turísticas en actividades sociales y culturales.

En resumen, se ha identificado que el PPM es un detonante en el desarrollo turístico económico en la comunidad, que a corto plazo permite generar proyectos innovadores de los productos y servicios turísticos, con el uso de la tecnología enfocada al desarrollo sostenible en la región del sureste zacatecano. Por ende, las conclusiones generan nuevas reflexiones como posibles líneas futuras de investigación en la metodología, con variables en la caracterización del desarrollo turístico económico y la combinación de otras metodologías en la evaluación para la permanencia del PPM según el contexto.

\section{Información complementaria}

\section{Agradecimientos}

Los autores reconocen a la Red Gestio y a la Red de Investigadores en Administración y Negocios, a través de la Dra. Luz del Carmen Morán Bravo, en la línea de investigación del turismo.

\section{Conflictos de interés}

Los autores del presente artículo establecen que no presentan conflicto de intereses en relación con la revista y sus editores. 


\section{Referencias}

Cayo-Velásquez, N. E. \& Apaza-Tarqui, A. (2017). Evaluación de la ciudad de Puno como destino turístico - Perú. Comuni@cción,8(2),116-124. https:// bit.ly/3vTfuKB

Consejo Nacional de Población [CONAPO]. (2020). Índice de marginación por municipio 2020. https://bit.ly/3aO5tVC

DATATUR. (2020). Glosario. Consultado el 3 de mayo de 2020. https://bit.ly/3EkzCta

Espeso-Molinero, P. (2019). Tendencias del turismo cultural. Revista de Turismo y Patrimonio Cultural, 17(6), 1101 1112. https://doi.org/10.25145/j.pasos.2019.17.076

Facchini, F. (2020). Cultura, diversidad cultural y desarrollo económico. Una perspectiva crítica de los trabajos recientes. Revista de Economía Institucional. 22(43), 79-117. DOl: https://doi. org/10.18601/01245996.v22n43.05

Fischer, L. \& Espejo, J. (2017). Introducción a la Investigación de mercados (6. ${ }^{\text {a ed.). }}$. McGraw Hill.

Flores-Pacheco, N. (2019). Desarrollo regional: motivo de participación en proyectos de turismo gastronómico. El caso de San Pablo Villa de Mitla, Oaxaca. Estud. soc. Rev. aliment. contemp. desarro. reg. [online]. Vol.29, N.53, e19685._https://doi.org/10.24836/ es.v29i53.685_

Hernández, R., Fernández, C. \& Baptista, M. del P. (2014). Metodología de la Investigación (6. a ed.). McGraw Hill.

Hofmann, A. (2015). Pueblos mágicos de la magia al desarrollo local. Buen Gobierno, (19), 106-120. https://bit.ly/3jGE5NS
Instituto Nacional de Estadística y Geografía [INEGI]. (2020). Panorama sociodemográfico de Zacatecas. Pinos, Zacatecas.47. https://bit.ly/3AQAd2N

Kotler, P. \& Armstrang, G. (2017). Fundamentos de Marketing. Pearson.

Orellana-Daube, D. F. (2020). El efecto global de la actual revolución tecnológica $4^{a}$ revolución industrial y la industria 4.0 en acción. Revista GEON (Gestión, Organizaciones Y Negocios), 7(2), 1-24. https://doi.org/10.22579/23463910.194

Organización Mundial del Turismo [OMT]. (2020). Innovation, Investments and Digital Transformation. https://bit. ly/3phVEI1

Parkin, M. (2018). Economía. Pearson.

Peniche, S., Laure, A. \& Cázares, L. (2017). El impacto ambiental del turismo internacional: caso de la huella de carbono de los vuelos internacionales hacia Puerto Vallarta, Jalisco, México. Investigaciones Turísticas, (14), 45-62. https://dx.doi.org/10.14461/INTURI2017.14.03

Pérez-Paredes, A., Cruz de los Ángeles, J. A., \& Torralba-Flores, A. (2017). Percepción de microempresarios poblanos ante el funcionamiento del Instituto Nacional del Emprendedor como política pública. Revista GEON (Gestión, Organizaciones Y Negocios), 4(1), 19-37.https://doi.org/10.22579/23463910.40

RDSTATION (2020). Marketing Digital. https://bit.ly/3BVTAsv

Rodríguez-Castellanos, A., \& San-Martin-Albizuri, N. (2020). Covid-19, globalización, complejidad e incertidumbre: algunas reflexiones sobre gestión empresarial en tiempos de crisis y más allá. Revista GEON (Gestión, Organiza- 
ciones Y Negocios), 7(2), 1-17. https:// doi.org/10.22579/23463910.219

Romero-P., Y, Navarro-J., E. \& Romero-Martínez, J. M. (2020). Destinos turísticos y capital creativo: El caso de la costa del sol en el sur de España. Revista de geografía Norte Grande, (77), 339-365. https://dx.doi.org/10.4067/ s0718-34022020000300339

Sandoval-Pillajo, L. L., Enríquez-Chugá, J. F., \& Sandoval-Pillajo, A. L. (2021). Las TIC en la educación y difusión de un producto turístico en Ibarra. Revista Conrado, 17(78), 291-296. Recuperado a partir de https://conrado.ucf.edu.cu/ index.php/conrado/article/view/1679

Secretaría de turismo [SECTUR], Secretaría de Turismo de Zacatecas [SECTURZ] \& Universidad Autónoma de
Zacatecas [UAZ]. (28 de abril del 2014). Estudio de competitividad de Pueblos Mágicos de Zacatecas. Municipio de Pinos, Zacatecas. SECTURZ. https://bit. ly/3pddQT4

Secretaría de Turismo [SECTUR]. (03 de enero del 2014). Pueblos Mágicos. Gobierno de México. https://bit. ly/3E3eQya

Secretaría de Turismo [SECTUR]. (2020). Programa Sectorial de Turismo 20202024. Gobierno de México. https://bit. ly/3pGgnFx

Torres-Flórez, D. (2020). La generación de valor entre las personas y las organizaciones. Revista GEON (Gestión, Organizaciones Y Negocios), 7(1), 4-8.https:// doi.org/10.22579/23463910.211 